\title{
Evidence of Anisotropic Reorientations of Water Molecules in the Cortex of the Rabbit Lens Detected by ${ }^{1}$ H-NMR Spectroscopy
}

\author{
1 Department of Biophysics, Medical Academy of Bydgoszcz, \\ ul. Jagiellonska 13, 85-067 Bydgoszcz, Poland \\ ${ }^{2}$ Institut für Organische Chemie der Freien Universität Berlin, \\ Takustr. 3, D-14195 Berlin, Germany
}

By J. Bodurka '* *, A. Gutsze ${ }^{1}$, G. Buntkowsky ${ }^{2}$ and H.-H. Limbach ${ }^{2}$

Dedicated to Prof. Dr. Dr. H. Pfeifer on the occasion of his 65th birthday

(Received April 10, 1994)

\section{${ }^{1} \mathrm{H}$ NMR / Rabbit lens / Residual magnetic dipole interaction / Anisotropic motion / Water ordering}

We studied the order of water molecules in different fragments of nucleus and cortex of lenses of 3 month old rabbits by investigating the ${ }^{1} \mathbf{H}$-NMR spin-spin relaxtion behaviour of the water protons at room temperature. The experiments were carried out using the Carr Purcell Meiboom Gill (CPMG) technique. The apparent relaxation rate was found to be dependent on the pulse spacing in the CPMG sequence in a different way for nucleus and cortex. While for the nucleus the pulse spacing dependence can be explained by chemical exchange of water protons, the pulse spacing dependence of the cortex protons suggests the existence of not fully averaged residual magnetic dipolar couplings among the protons of a water molecule. To support this interpretation, measurements of the ratio of the solid echo $\left(90_{x}-90_{y}\right)$ to the Hahn echo $\left(90_{x}-180_{x}\right)$ amplitude were carried out for the same samples. These experiments give the expected ratio of $K=0.5$, characteristic of no residual couplings for the nucleus, but a ratio of $K=0.7$ for the cortex, which is characteristic of residual dipolar couplings, caused by anisotropic reorientations of the water molecules. Thus, evidence for an ordered state of the water molecules in the cortex has been found.

In dieser Arbeit wird die transversale Relaxation der Wasserprotonen in verschiedenen Teilen von Nukleus und Cortex der Augenlinsen drei Monate alter Kaninchen 'H-NMRspektroskopisch mit Hilfe der Carr-Purcell-Meiboom-Gill (CPMG) Technik untersucht.

* For correspondence. 
Daraus ergeben sich Informationen über den Ordnungsgrad des Wassers in den verschiedenen Umgebungen. Die beobachteten Relaxationsraten von Nukleus und Cortex hängen in unterschiedlicher Weise vom Pulsabstand ab. Während die Abhängigkeit im Fall des Nukleus mit Protonen- und Wasseraustauschprozessen zwischen unterschiedlichen chemischen Umgebungen im Einklang steht, läßt sie sich im Fall des Cortex nur durch eine nicht ausgemittelte magnetische Dipol-Dipol-Kopplung der Wasserprotonen erklären. Zur Verifizierung dieser Interpretation wurden zusätzlich die Amplituden von „Festkörperechos" nach $90_{x}^{\circ}-\tau-90_{y}^{\circ}$-Anregung und von „Hahn-Echos“ nach $90_{x}^{\circ}-\tau-180_{y}^{\circ}$-Anregung vermessen. Das Echoverhältnis $\mathrm{K}=0.5$ für den Nukleus steht mit einer Ausmittlung der dipolaren Wechselwirkungen im Einklang, während der Wert von $\mathrm{K}=0.7 \mathrm{im}$ Fall des Cortex die Beobachtung der nicht ausgemittelten dipolaren Wechselwirkungen unterstützt. Dieses Phänomen läßt sich mit einer anisotropen Rotationsdiffusion der Wassermoleküle im Cortex erklären, die durch einen von Null verschiedenen Ordnungsgrad bedingt ist.

\section{Introduction}

The magnetic relaxation of protons in water molecules of heterogenous and biological materials have been the subject of increasing interest. Especially the rapid progress in medical application of NMR has caused needs for a detailed understanding of proton relaxation processes in biological systems $[1-4]$. The importance of the investigation of water behaviour in biological systems results from the fact, that water is the primary biological solvent in which all other cellular components and solutes diffuse and interact with one another. In many biochemical reactions water serves as the donor of hydrogen ions, and the dissociation of water contributes to the regulation of the intracellular $\mathrm{pH}$. Because of its high heat capacity, water stores metabolic energy and helps to regulate the temperature of an organism. Through hydrophobic and hydrophylic interactions water contributes to the stabilization of the three-dimensional macromolecular structure, and even stabilizes giant cellular components such as lipid bilayer membranes and microtubules. Finally, water is also one of the primary end products of all metabolic pathways, so, cells do not only use water, they also synthesize it. Therefore, a great deal of biological functions pertain to the consumption, regulation, and excretion of water $[5,6]$. The intracellular compartments influence the behaviour of hydratation water and, via the material exchange process, also the bulk water. Therefore, relaxation properties of water in such systems have to reflect the relaxation behaviour of the whole system. Numerous experimental studies conducted on the physical state of intracellular water indicate that intracellular water and hydrated intracellular ions exist in a highly ordered state [7-9]. In the present work we give evidence that the motion of water in the rabbit lens is restricted, in other words, there exists some kind of water ordering. This conclusion is based on proton relaxation measurements.

The mammalian lens has a relatively simple and regular structure. It has the shape of a flattened globe. It is surrounded by a capsule, under which 
there is a layer of epithelial cells. These cells reproduce and create elongated lens fibers. They are formed through life, and new fibers cover the old one. The oldest fibers in the center of the lens are glued together, and form a compact mass known as the nucleus. The rest of the lens forms the cortex. The fibers make up the bulk of the lens cortex and nucleus. Each fiber represents an elongated cell with membrane. They loose their nuclei as they grow older [10]. A relatively high degree of physico-chemical and structural organisation is needed to achieve the transparency of the lens. The normal eye lens contains approximatly $65 \%$ of water and $35 \%$ of organic materials, which are mainly structural proteins. With this high protein content $(33 \%$ of the total weight), the lens has the highest protein concentration of all the organs in the body $[10,11]$. About $85 \%$ of the total mass of proteins form crystallins, called: $\alpha\left(15 \%, \mathrm{mw} \approx 10^{6} \mathrm{D}\right), \beta\left(55 \%, \mathrm{mw} \approx 4 \times 10^{4} \mathrm{D}\right)$, and $\gamma\left(15 \%, \mathrm{mw} \approx 2 \times 10^{4} \mathrm{D}\right)$.

In general, protons in water protein systems may be classified into at least three different phases, according to the physico-chemical properties of their environment. These proton phases are: Proteins, hydration (nonfreezable, bound) water, and bulk (freezable, free) water. The reorientation of water molecules belonging to the free water is assumed to be isotropic, which means that the motion of the water molecules can be described by a single correlation time. Its dynamic behaviour is analogous to pure water, and there is no water ordering in that phase. Compared to this, restrictions for the possible motions of water molecules belonging to the bound phase may exist, caused by the interaction with the neighbouring protein molecule. In particular, the average overall motion of water molecules in the bound phase is reduced, and therefore the residence time of water molecules in a given volume element may be increased. Furthermore, molecular reorientations of water molecules may become anisotropic, for example caused by hydrogen bonds to protein. Evidence for these kinds of reduced freedom of mobility has been reported on the level of a macromolecular model of the solutions, for tissues and even for the whole organism [12-14].

Between the different phases of water/protein systems two kinds of exchange processes, visible by ${ }^{1} \mathrm{H}-\mathrm{NMR}$, may exist. The first kind of exchange process is the material exchange of a proton or a protonated group by chemical exchange or the exchange of whole molecules (in which protons are located, e. g. mainly water molecules) by diffusion-like processes. In the second kind of exchange processes only the magnetization of different protons is exchanged, i. e. there is an (immaterial) spin exchange, which is also known as cross-relaxation or spin diffusion $[15,16]$. Most measurements, performed on water protein solutions so far, were done on systems with - compared to the eye lens - relatively low protein concentrations. For such systems the average fraction of bound water is less than $5 \%$ of the total water content $[17-19]$. The relaxation behavior of the water protons 
in such a protein solution was explained by the assumption of a fast proton protons exchange between these two phases [20], and single spin-lattice $\left(T_{1}\right)$, and spin-spin $\left(T_{2}\right)$ relaxation times are observed for such systems, i. e.:

$$
\frac{1}{T_{k}}=\sum_{\mathrm{i}} \frac{P_{i}}{T_{k i}}, \quad k=1,2 .
$$

Moreover, because of the high amount of free water $\left(p_{f}>95 \%\right)$, the relaxation behavior is determined by the relaxation of the free water, and the resulting average relaxation rates $T_{\mathrm{k}}^{-1}(k=1,2)$ are practically the relaxation rates of the free water, which are in the order of the rates of pure water. The experimentally found increase of the relaxation rates of water in protein solutions, compared to the rates of pure water, may arise from hydrodynamic effects or exchange processes [20,21]. Furthermore, the relaxation rates should show the normal liquid-like behaviour, i. e. $T_{2} \approx T_{1}$, which means, the ratio $R=T_{1} / T_{2}$ should be close to 1 as is the case for pure water $[22,23]$. Comparing these results of water relaxation in water protein solutions with low protein concentrations to the relaxation of water in the mammalian lens, characteristic differences are found [24-26]. There is a single spin-lattice relaxation time $\left(T_{1}\right)$ but two different spin-spin relaxation times $\left(T_{21}, T_{22}\right)$. Moreover, the ratio $R=T_{1} / T_{2}$ is far from unity, i. e. $T_{21}$, $T_{22} \ll T_{1}$, exhibiting a more solid-like behaviour of the water in the lense [23]. To explain the relaxation properties of the water in the rabbit lens the following model was proposed [26]. The exchange rate between free and bound water molecules is fast on the time scale determined by $T_{1}$ (order of seconds) but slow on the time scale determined by $T_{2}$ (order of milliseconds). Therefore, only the $T_{1}$ times are averaged by the exchange process. To explain the strong difference between spin-spin and spin-lattice relaxation rates one may either assume a broad distribution of motional correlation times or one may, alternatively, assume that the reorientations of the water molecules in the lens are anisotropic [23, 27]. In order to reproduce the observation that $T_{2} \ll T_{1}$ one has, however, to assume an unrealistically large distribution of correlation times of the water orientation $[23,28]$. Therefore, the second possibility, i. e. anisotropic reorientations of the water molecules, which results in incomplete averaging of the dipolar interactions, has to be examined more closely. It is the aim of this paper to give experimental evidence for the possible existence of residual dipolar couplings between protons of water molecules as the result of anisotropic reorientations caused by water ordering in the rabbit lens.

\section{Materials and Methods}

The measurements were done on fragments of the rabbit lenses from 5 different 3 month old animals. The fragments were prepared in such a way 


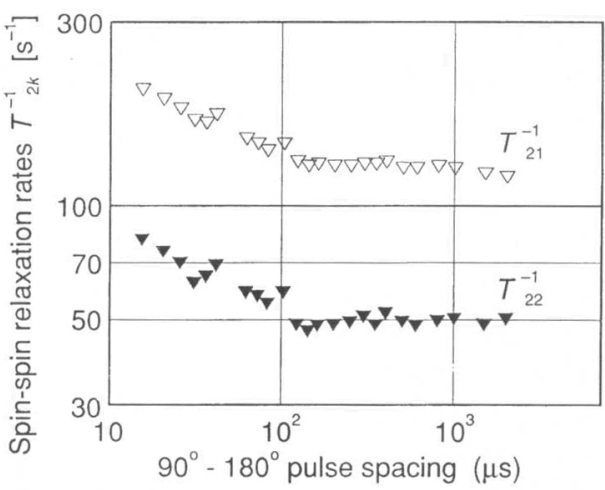

Fig. 1. Apparent transverse relaxation rates for the rabbit lens fragment containing tissues of both nucleus and cortex, plotted against pulse spacing ( $t_{\mathrm{CPMG}}$ ) in the CPMG experiment. The fragment was obtained as a cross-section through the whole rabbit lens. Measurement $300 \mathrm{MHz}, 298 \mathrm{~K}(\nabla) T_{21}^{-1},(\nabla) T_{22}^{-1}$.

that they either contained only tissue from the cortex or nucleus of the lense, or tissues of both nucleus and cortex. The latter fragments where obtained as a cross-section through the whole rabbit lens. The spin-spin relaxation times $T_{2}$ were measured with the Carr-Purcell-Meiboom-Gill (CPMG) sequence [29] as a function of the pulse spacing, $t_{\mathrm{CPMG}}$, between the pulses in the sequence. To reduce the $B_{1}$ field inhomogeneity and pulse width errors [30] only the even echoes were registered. The relaxation functions were analysed by the non-linear least squares method (Marquart algorithm) [31]. We observed a biexponential decay of the echo amplitudes, characterized by the two time constants $T_{21}, T_{22}$. Additionally, we measured for each sample also the echo maximum, $\mathrm{A}(\mathrm{SE})$, for the solid echo sequence $\left(\left(90^{\circ}\right)_{\mathrm{X}}, t_{\mathrm{SE}},\left(90^{\circ}\right)_{\mathrm{Y}}\right)$, and the echo maximum, $\mathrm{A}(\mathrm{HE})$, of the Hahn echo sequence $\left(\left(90^{\circ}\right)_{\mathrm{X}}, t_{\mathrm{HE}},\left(180^{\circ}\right)_{\mathrm{X}}\right)$ as a function of the pulse spacing time $t=t_{\mathrm{SE}}=t_{\mathrm{HE}}$, and calculated their ratio $K=\mathrm{A}(\mathrm{SE}) / \mathrm{A}(\mathrm{HE})$. The values for $t_{\mathrm{CPMG}}$ and $t_{\mathrm{SE}(\mathrm{HE})}$ ranged from 20 microseconds to 4 milliseconds, and 20 microseconds to 35 milliseconds, respectively. All measurements were done on a BRUKER MSL 300 spectrometer working at $300 \mathrm{MHz}$. The typical length of a $\left(90^{\circ}\right)$ pulse was 10 microseconds. The measurements were performed at room temperature $(298 \mathrm{~K})$.

\section{Results and Discussion}

The measurements of the pulse spacing dependence of the apparent spinspin relaxation rate $T_{2}^{-1}$, determined by the CPMG sequence, are shown in Figs. 1 to 3 . Figure 1 shows the data from lens fragments containing both 


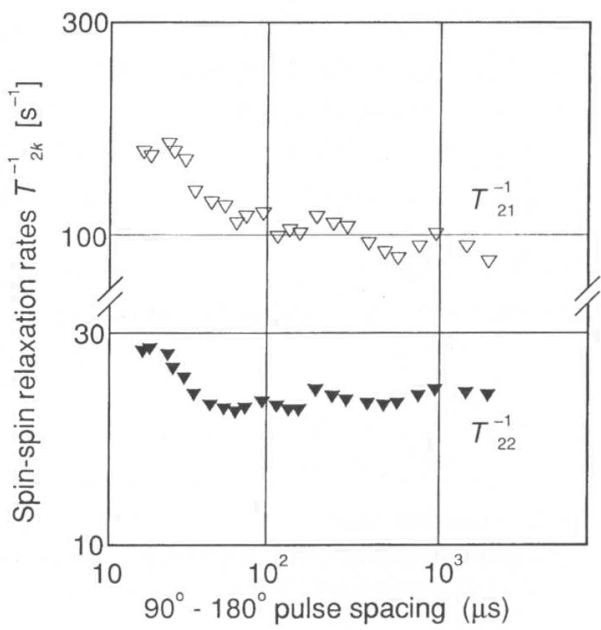

Fig. 2. Same as Fig. 1 for the rabbit lens fragment containing only tissue from the cortex.

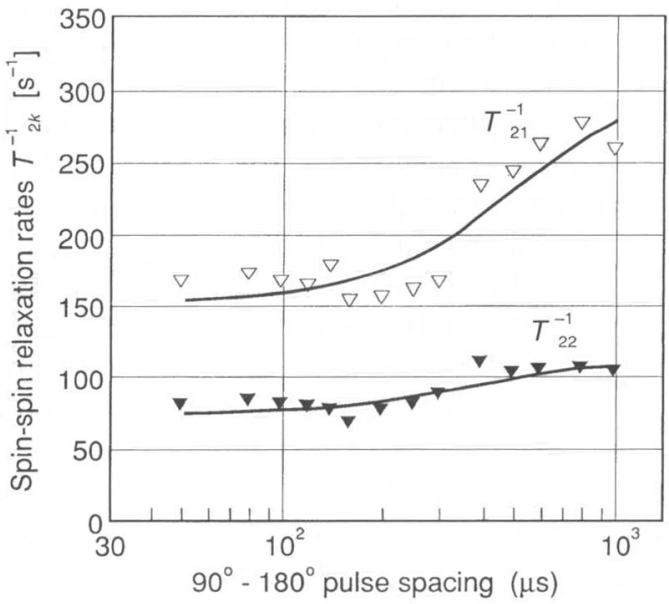

Fig. 3. Same as Fig. 1 for the rabbit lens fragment containing only tissue from the nucleus. The fit of the experimental data in terms of the Luz-Meiboom expression [38] is shown as a solid line.

cortex and nucleus, and Fig. 2 shows the results of the cortex fragments alone. For the whole range of interpulse time spacings we could describe the spin-spin relaxation function very well as a bi-exponential decay, using two transverse relaxation rates $T_{21}^{-1}, T_{22}^{-1}$. Both rate constants exhibit the same dependence on the pulse spacing. The spin-spin relaxation rates decreases, if the time $t_{\text {CPMG }}$ between the pulses is increased, Figure 3 shows 
the results of the same experiment, performed on fragments of the nucleus. It exhibits interesting differences to the previous experiment, because for the nucleus the transverse relaxation rates increase if the time between the pulses is increased. Comparing these results to experiments on other water protein solutions (WPS), for example bovine serum albumine (BSA) solutions, we find that these solutions exhibit the same behaviour as the nucleus, i. e. with decreasing pulse spacing $t_{\mathrm{CPMG}}$ an increase of the $T_{2}$ was observed [17]. According to the literature, the dependence of the transverse relaxation rates, measured with the CPMG sequence, on the pulse repetition rate are affected by several factors:

1) diffusion through static field inhomogeneities [32]

2) spin locking effects (shift from $T_{2}$ measurements to $T_{1}$ measurement when $t_{\text {CPMG }}$ becomes very short) [33] 35]

3) chemical exchange between sites with different chemical shifts [34,

4) existence of a non zero time average of dipolar energy of the spin pair within water molecules as a result of anisotropic reorientation $[12,36]$.

To examine, whether the first case, i. e. diffusion through field inhomogeneities, can significantly contribute to the relaxation times in our experiments, we calculated their effects in the following way: The size of the static field inhomogeneities, $\Delta B_{0}$, can be estimated from the half width of the NMR line of a sample of pure water, because its homogeneous width is negligibly. The self diffusion coefficient of the water, $D$, in the rabbit lens is $10^{-9}\left[\mathrm{~m}^{2} / \mathrm{sec}\right]$ [4]. Now, we estimate the contributions to the transverse relaxation rate from diffusion $\left(T_{2 \mathrm{Diff}}^{-1}\right)$ through static field inhomogeneities [32] to the observed relaxation rates, using the equation:

$$
T_{2 \text { Diff }}^{-1}=1 / 3\left(\gamma^{2} \Delta B_{0} D t_{\text {CPMG }}^{2}\right) \text {. }
$$

The result of this estimation is, that $T_{2 \mathrm{Diff}}^{1}$ is about two orders in magnitude smaller than the observed relaxation rates at $t_{\mathrm{CPMG}} \rightarrow 0$. Therefore, we can exclude this factor as an explanation for the observed results. Spin locking effects can also be of only minor importance to the relaxation behavior, because they should influence the results in the same way for nucleus and cortex, in contrast to the experimental findings. Therefore, we can concentrate on the last two explanations.

The experimental results presented in Figs. 2 and 1, which were obtained for the cortex of the lens and fragments containing cross-sections of the whole lens (i. e. cortex and nucleus simultaneously), show a decrease of the measured $T_{2}$ as a function of the pulse spacing. This dependence on the pulse spacing can be explained by anisotropic reorientations of the water molecules, which result in a nonvanishing residual magnetic dipolar interaction among the protons of a water molecule $[12,37]$. Following the calculations given in [12] we can estimate this residual coupling to be in the range of $10-30 \mathrm{sec}^{-1}$. Furthermore, as discussed in [12], the ratio $K=$ 


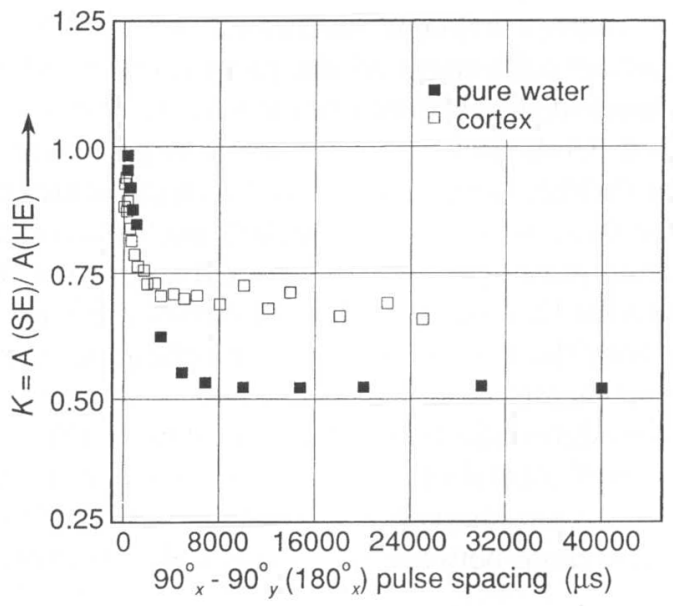

Fig. 4. The ratio $K=\mathrm{A}(\mathrm{SE}) / \mathrm{A}(\mathrm{HE})$ of the solid echo $\left(90_{x}-90_{y}\right)$ to the Hahn echo $\left(90_{x}-\right.$ $180_{x}$ ) amplitude (A(SE), A(HE), respectively) for the rabbit lens fragment containing only tissue from the cortex and for pure water, plotted against pulse spacing ( $t_{\mathrm{SE}}, t_{\mathrm{HE}}$ respectively). Measurement $300 \mathrm{MHz}, 298 \mathrm{~K}$, (口) pure water, ( $\square$ ) cortex.

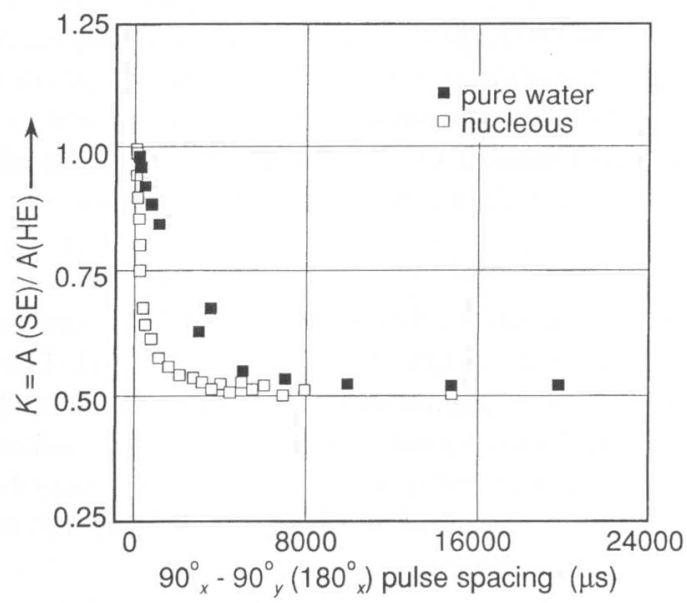

Fig. 5. Same as Fig. 4 for the rabbit lens fragment containing only tissue from the nucleus and for pure water. $(\square)$ pure water, $(\square)$ nucleus.

$\mathrm{A}(\mathrm{SE}) / \mathrm{A}(\mathrm{HE})$ is equal to 0.5 in the case of vanishing residual dipolar interaction (ordinary liquids), but greater if the dipolar interactions are not fully averaged. If we compare these predictions with Fig. 4 and Fig. 5 we find for the cortex a value of $K=0.7$, while for the nucleus as well as for pure 
water, which we measured as control experiment, a value of $K=0.5$ is found. Thus we conclude, that the observed behaviour can be explained by motional restrictions for the water molecules in the cortex, which lead to a non averaged dipolar interaction.

Figure 3 shows the results of the pulse spacing dependence of $T_{2}$ measured with the CPMG sequence for the nucleus. The dependence of $T_{2 \mathrm{k}}$ $(k=1,2)$ versus $t_{\mathrm{CPMG}}$ has the opposite tendency as compared to the data presented in Figs. 1 and 2, i. e. the rate increases with the pulse spacing. These results are similar to other results obtained previously [17] for water protein solutions, which have been explained by chemical exchange [34, 35] and have been shown to obey the Luz-Meiboom expression [38]. The fit of the experimental data in terms of this expression is shown as a solid line in Figure 3, which reproduces the main tendency of the experimental data. Thus, in contrast to the cortex, water in the nucleus behaves as an ordinary liquid, and any possible motional restrictions, as discussed for the cortex, can be of only minor importance. In other words, the dynamic characteristics of water in the nucleus are more close to water in protein solutions. To summarize, our results suggest that for the cortex there exists some residual magnetic dipolar interaction between the two adjacent protons in a water molecule, while this interaction is absent for the nucleus. Since the dipolar interaction has no isotropic value, which would only survive if the water molecules undergo fast isotropic reorientations, we can furthermore conclude, that there is an anisotropic distribution of the water molecules in the cortex, in other words, the water is ordered. Similar kinds of water ordering have already been observed, for example in lyotropic liquid crystals [39], caused by the regular structure of the liquid crystal. Thus, by our experiments, we can conclude, that there exists a regular structure of the lens proteins, leading to a supramolecular organisation of the proteins [40], which induces the water ordering in the lens [8]. This ordering then causes restrictions on the reorientational motions, which finally result in the residual magnetic dipole interaction between the protons of water molecules in these oriented phases. The presence of such anisotropic reorientations depends strongly on the degree of order. Since our experiments suggest, that the anisotropic reorientations of the water molecules in the cortex are absent in the nucleus, we can furthermore conclude, that the proteins in the cortex are in a highly ordered state, while the proteins in the nucleus show no order. Thus, we can confirm findings from previous investigations, which suggested a high degree of short range order [8] for the cortex, but practically no short range order for the nucleus [41]. The question now arises, what are the biological reasons for this kind of water ordering in the cortex. Obviously, any residual magnetic dipolar interaction by itself is of no direct importance for the physico-chemical processes taking place in the cell. Therefore, it can only be a side product of some other cause. As has been shown by light scattering [42], the transparency of the 
lens is maximal if there is a short range order of the lens proteins. So, finally we can say that the water ordering, which we have observed in the lens, is caused by its most fundamental function, to be transparent for light.

\section{Acknowledgement}

This work was supported by research KBN programme 402689101 .

\section{References}

1. P. A. Bottomley, T. H. Foster, R. E. Argersinger and L. M. Pfeifer; Med. Phys. 11(4) (1984) 425-447.

2. P. Mansfield and P. G. Moris, NMR Imaging in Biomedicine, Academic Press, New York (1982).

3. R. Damadian, NMR in Medicine, Springer-Verlag New York (1981).

4. P. T. Beall, S. R. Amtey and S. R. Kasturi, NMR Data Handbook for Biomedical Sciences, Pergamon Press New York (1984).

5. F. Franks, Water: A Comprehensive Treatise, vols 1-6, Plenum Press, New York (1972-1979).

6. F. H. Stillinger, Water revisited. Science, 209 (1980) 451-457.

7. G. N. Ling, Physiol. Chem. Phys. Med. NMR, 15 (1983) 137-154.

8. T. Glonek and J. V. Greiner, Ophtalmic Res. Res. 22 (1990) 302-309.

9. E. C. Trantham, H. E. Rorschach, J. S. Clegg, C. F. Hazlewood, R. M. Nicklow and N. Wakabayashi, Biophys. J. 45 (1984) 927-938.

10. A. Moses, Adler's Physiology of the eye, The C. V. Mosby Company London (1981).

11. H. Maisel, The Ocular Lens, Marcel Dekker Inc. New York (1985).

12. J. P. Cohen-Addad and R. Vogin, Phys. Rev. Letters 33 (1974) 940-943.

13. S. Peto, P. Gillis and V. P. Henri, Biophys. J. 57 (1990) 71-84.

14. J. C. Wu, E. C. Wong, E. L. Arrindell, K. B. Simons, A. Jesmianowicz and J. Hyde, Invest. Ophthalmol. \& Vis. Sci. 34 (1993) 2151-2158.

15. J. R. Zimmermann and W. E. Brittin, J. Chem. Phys. 61 (1957) 1328-1333.

16. H. Winkler and D. Michel, Adv. in Coll. and Interf. Sci. 23 (1985) 149-179.

17. B. P. Hills, S. F. Takaces and P. S. Belton, Molec. Physics. 67 (1989) 903-918.

18. D. Grucker, J. Steibel, Y. Mauss, B. Dumitresco, J. P. Armspach and J. Chambron, Molec. Phys. 70 (no 5) (1990) 903-919.

19. J. Zhong, J. C. Gore and I. M. Armitage, Magn. Res. Med. 11 (1989) 295-308.

20. R. Kimmich, W. Nusser and T. Gneitting, Colloids and Surfaces 45 (1990) 283302.

21. S. H. Koenig and R. D. Brown, CRC Press, Boca Raton, Fl. 2 (1987) 75-114.

22. N. Bloembergen, E. M. Purcell and R. V. Pound, Phys. Rev. 73 (1948) 679-712.

23. H. Pfeifer, NMR Basic Principles and Progres vol 7 (1972) 55-153.

24. P. J. Stankiewicz, K. R. Metz, J. W. Sassani and R. Brigs, Invest Ophthalmol. Vision Sci. 30 (1989) 2361-2369.

25. A. Gutsze, D. Deninger, R. Olechnowicz and J. A. Bodurka, Lens \& Eye Tox. Res. $8(2 \& 3)(1991) 155-163$.

26. A. Gutsze, J. A. Bodurka, R. Olechnowicz and A. Jesmianowicz, Colloids and Surfaces A: Physicochem. and Engineering Aspects 72 (1993) 295-299.

27. H. A. Resing, Advan. Molec. Relaxation Processes, 3 (1972) 199-226.

28. J. Bodurka, A. Gutsze and R. Olechnowicz, Proc. XXV Polish NMR Symposium, Report nr 1616/PL, Krakow 1993 (1993) 22-36.

29. S. Meiboom and D. Gill, Rev. Sci. Instrum. 29 (1958) 688-691.

30. M. H. Levit and R. Freeman, J. Magn. Res. 43 (1981) 65-80. 
31. P. R. Bevington, Data Reduction and Error Analysis for Physical Sciences, McGraw Hill, New York (1969).

32. H. Y. Carr and E. M. Purcell, Phys. Rev. 94 (1954) 630-638.

33. G. E. Santyr, R. M. Henkelmann and M. J. Bronskill, J. Magn. Reson. 79 (1988) $28-44$.

34. A. Allehard and H. S. Gutowsky, J. Chem. Phys. 41 (1964) 2115-2126.

35. A. Allehard and H. S. Gutowsky, J. Chem. Phys. 42 (1965) 1587-1599.

36. S. B. Ahmad, K. J. Packer and J. M. Ramsden, Molec. Phys. 33 (1977) 857-874.

37. J. P. Cohen-Addad, J. Chem. Phys. 60 (no 6) (1974) 2440-2453.

38. Z. Luz and S. Meiboom, J. Chem. Phys. 39 (1963) 438.

39. M. Bloom, E. E. Burnell, S. B. Roeder and M. Valic, J. Chem. Phys. 66 (no 7) (1977) 3012-3020.

40. F. A. Bettelheim, Physical basis of lens transparency. In the Ocular Lens-Structure, Function, and Pathology. Marcel Dekker New York (1985) 265-300.

41. H. Wang and F. Bettelheim, Exp. Eye Res. 55 (1992) 387-391.

42. M. Delay, A. Tardieu, Nature 302 (1983) 415-417. 